\title{
Preeclampsia and Its Complications Exacerbate Development of Postpartum Depression: A Retrospective Cohort Study
}

\author{
Ying Ye $\mathbb{D}^{1,2}$ Li Chen, ${ }^{3}$ Jiani Xu $\mathbb{D},{ }^{1,2}$ Qinjin Dai $\mathbb{D},{ }^{4}$ Xin Luo, ${ }^{1,2}$ Nan Shan $\mathbb{D},{ }^{1,2}$ \\ and Hongbo Qi $\mathbb{i}^{1,2}$ \\ ${ }^{1}$ The Department of Obstetrics, The First Affiliated Hospital of Chongqing Medical University, Chongqing 400016, China \\ ${ }^{2}$ State Key Laboratory of Maternal and Fetal Medicine of Chongqing Municipality, Chongqing Medical University, \\ Chongqing 400016, China \\ ${ }^{3}$ The Bishan Hospital of Chongqing, Chongqing 402760, China \\ ${ }^{4}$ Guangzhou Women and Children's Medical Center, Guangzhou Medical University, Guangdong 510623, China
}

Correspondence should be addressed to Nan Shan; shannancqmu@163.com and Hongbo Qi; qihongbocqmu@163.com

Received 26 December 2020; Revised 9 April 2021; Accepted 15 April 2021; Published 23 April 2021

Academic Editor: A.Seval Ozgu-Erdinc

Copyright $(2021$ Ying Ye et al. This is an open access article distributed under the Creative Commons Attribution License, which permits unrestricted use, distribution, and reproduction in any medium, provided the original work is properly cited.

\begin{abstract}
Background. Hypertensive disorders were proved to be associated with the development of depression. But it is unclear if pregnancy-induced hypertensive diseases, especially preeclampsia (PE), will affect postpartum moods. We aimed to determine the incidence rate of postpartum depression (PPD) in PE patients and comprehensively evaluate the association between PPD and PE, including its severity and complications. Methods. 425 participants including $130 \mathrm{PE}$ mothers were enrolled in this retrospective cohort study. Each woman was asked to complete a questionnaire integrating the Edinburgh Postnatal Depression Scale (EPDS), the Leakage Index Questionnaire, and a pain scale questionnaire within 6 weeks after delivery. The EPDS cut-off score above 13 was recognized as screening positive for PPD. Data between groups were compared by bivariate analysis. Results. PE mothers showed a direct tendency to PPD development. The positive screening for PPD in the PE group was significantly higher than that of the control group (30.77\% vs. $14.58 \%)$. Based on the results of the regression model, women diagnosed with severe PE and fetal growth restriction were more inclined to develop PPD than normal ones (AOR: 2.759, 95\% CI: 1.206-6.315 and AOR: 3.450, 95\% CI: 1.596-7.458). It is also indicated that postpartum pain exacerbated the odds of PPD in PE patients (AOR: 1.509, 95\% CI: 1.078-2.114). Conclusions. PE was an independent risk factor for PPD. Its severity and complications exacerbate the development of PPD. Doctors and society should pay more attention to PE patients after delivery against the development of PPD.
\end{abstract}

\section{Introduction}

Postpartum depression (PPD) is a major depressive episode that begins within 6 weeks after delivery [1]. PPD affects a significant number of mothers, as the global prevalence of PPD reportedly ranges from $3 \%$ to $38 \%[2,3]$. Mothers with PPD often exhibit sadness, loss of interest and joy, feelings of helplessness, difficulty concentrating and remembering, and sleep disturbances. PPD may negatively impact maternal health, parenting, and subsequently the development of children. It may result in abusive parenting, maternal suicide, and infanticide $[4,5]$. Besides, it can lead to negative sequelae for the off- spring, including delayed cognitive development, behavioral problems, and even suicidal ideation [6-8]. Therefore, identifying the risk factors for PPD is important for earlier detection and prevention of negative consequences of PPD.

This disorder may be caused by multiple risk factors, including the history of depression, preterm delivery, poor marital relationship, and low social income $[9,10]$. These factors have been fully elucidated to be associated with PPD, and recently, a few studies have evaluated the effects of pregnant complications on PPD, such as preeclampsia (PE).

As reported, hypertensive disorder in pregnancy (HDP) is a risk factor for depression, and the prevalence 
is about $20 \%-30 \%$ [11]. PE is one HDP characterized as hypertension developing after 20 weeks of gestation with the coexistence of $\geq 1$ of a new onset of (1) proteinuria, (2) maternal organ dysfunction, or (3) uteroplacental dysfunction [12]. PE is one of the leading causes of maternal/fetal mortality and morbidity worldwide and is responsible for around 60,000 deaths [13]. PE directly threatens mothers and causes various adverse fetal outcomes, leading to small-for-gestational-age babies, premature delivery, and infant death [14].

Our previous study demonstrated that PE patients had nearly 3-fold increased odds for PPD compared to normal women, and patients with severe PE had a more than 4-fold higher risk of screening positive for PPD [15]. However, whether the severity of PE and fetal outcomes would contribute to PPD has not been investigated.

Herein, we aimed to compare the incidence rate of PPD in PE and normal women by employing the EPDS and to comprehensively evaluate the association between PPD and $\mathrm{PE}$, especially its severity and complications. In addition, it has been previously reported that pelvic floor symptoms, urinary incontinence, and pain would affect postpartum moods $[16,17]$, so we also employed the Leakage Index Questionnaire and the pain scale.

\section{Methods}

2.1. Study Design and Participants. In this two-center retrospective cohort study, patients who delivered between October 1, 2018, and August 30, 2019, were enrolled from the First Affiliated Hospital of Chongqing Medical University and Qinghai Red Cross Hospital. All patients were asked to independently complete the questionnaires within about 6 weeks after delivery. With informed consent, the answers of the patients can be used here.

2.2. Inclusion and Exclusion Criteria. The inclusion criteria were (1) diagnosis of PE by elevated blood pressure (systolic pressure $\geq 140 \mathrm{mmHg}$ or diastolic pressure $\geq 90$ and with proteinuria) after 20 weeks of gestational age according to the ACOG guidelines (2019), (2) diagnosed with PE accompanied with fetal growth restriction (FGR), (3) maternal age ranging from 18 to 45 years, and (4) gestational age $\geq 28$ weeks.

The exclusion criteria were as follows: (1) presence of other complications, such as gestational diabetes mellitus, intrahepatic cholestasis of pregnancy, and hyperthyroidism; (2) preterm (gestational age less than $36^{+6}$ weeks) not caused by PE; (3) preexisting mental diseases, history of depression; and (4) stillbirth or giving birth to a malformed fetus (including any minor anomalies).

2.3. Measurements. All details of maternal and neonatal conditions during pregnancy and delivery were obtained from the hospital information systems. After applying the inclusion and exclusion criteria, we invited mothers for a clinical visit within 6 weeks after delivery and encouraged them to participate in our questionnaires, including EPDS, Leakage
Index Questionnaire, and pain scale (numerical rating scales).

EPDS is the most commonly used PPD scale worldwide and is one of the most authoritative self-evaluation scales to screen for PPD [18]. Each of its 10 items is divided into 4 grades and scored from 0 to 3 . The total score ranges from 0 to 30 , with higher scores signifying more serious PPD [19]. Compared to other questionnaires, it has a satisfactory diagnostic efficiency and is more concise to subjects [20]. The sensitivity of EPDS has been proven to range from 0.67 to 1.00 , and the specificity is consistently 0.87 or higher when the cut-off value is 13 [21]. Therefore, a score of EPDS $\geq 13$ was determined to be positive for PPD screening in our study.

The Leakage Index Questionnaire (involving 3 items with multiple choices) and pain scale were used to evaluate the recovery of muscles in the pelvic floor and the degree of postpartum pain in mothers, respectively. The scores of the former range from 0 to 6 and from 0 to 10 in the latter. The higher scores on the Leakage Index Questionnaire predict poorer recovery of pelvic floor muscles. Moreover, educational background, annual family income, and milk feeding methods were also investigated in our questionnaire (details are shown in Figure S1).

Besides, severe PE was diagnosed as (1) systolic pressure $\geq 160 \mathrm{mmHg}$ or diastolic pressure $\geq 110 \mathrm{mmHg}$, measured at least every 4 hours; (2) platelet count $\leq 100 *$ $10^{9} / \mathrm{L}$; (3) abnormal liver function (elevated liver enzymes twice the upper limit) without other diseases; (4) renal dysfunction $(\mathrm{Scr} \geq 97.24 \mu \mathrm{mol} / \mathrm{L})$ in the absence of other diseases; (5) pneumonedema; (6) new-onset headache without other diseases; and (7) blurred vision [22]. FGR was defined as an infant birthweight below the $10^{\text {th }}$ percentile of the average of infants at the same gestational age. Patients without any complications during the perinatal period were eligible for enrolment in the normal group.

2.4. Statistical Analysis. This study was designed to detect a $15 \%$ absolute difference between groups with $90 \%$ power and a $5 \%$ type I error rate. We assume that the incidence of PPD was about $30 \%$ in the PE group and $15 \%$ in the control group. Therefore, a sample size of 380 (88 in the PE group and 292 in the control group) was needed. The MedSci Sample Size Tools (MSST, version 5.7.15, copyright 2020 MedSci.cn) were applied for calculating. We recruited $130 \mathrm{PE}$ patients and 295 healthy women.

Variables following normal distribution were compared via an independent $t$-test and presented as the mean \pm standard deviation. Otherwise, variables were described as the mean \pm quartile and examined by the Kruskal-Wallis test. Differences in the classified variables were evaluated by the chi-squared test. $P<0.05$ was considered significant. A multivariate logistic regression model was used to evaluate adjusted odds ratios. Confounding factors include age, BMI, gestational days, baby weight, delivery model, Leakage Index Score, milk-feeding ways, and pain scale, which were previously reported to be connected with PPD or unmatched between PE and normal groups. All statistical analyses were conducted on SPSS 23.0 (SPSS Inc, Chicago, USA). 
TABLE 1: Comparison of baseline characteristics between normal and PE women.

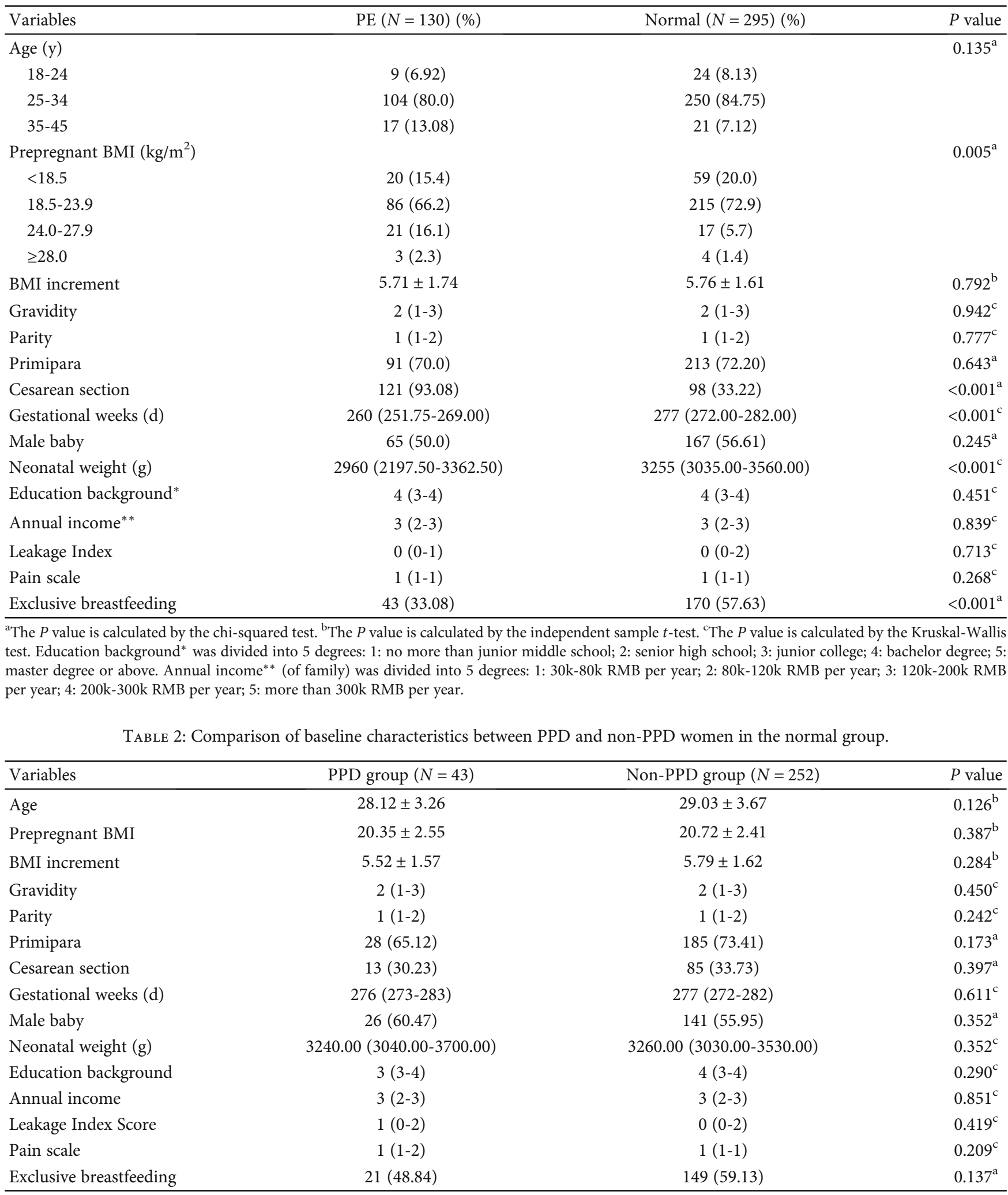

${ }^{a}$ The $P$ value is calculated by the chi-squared test. ${ }^{b}$ The $P$ value is calculated by an independent sample $t$-test. ${ }^{c}$ The $P$ value is calculated by the Kruskal-Wallis test. 
TABLe 3: Comparison of baseline characteristics between PPD and Non-PPD women in the PE group.

\begin{tabular}{|c|c|c|c|}
\hline Variables & PPD group $(N=40)(\%)$ & Non-PPD group $(N=90)(\%)$ & $P$ value \\
\hline Severe PE & $16(40.0)$ & $30(33.3)$ & $0.295^{\mathrm{a}}$ \\
\hline FGR & $17(42.5)$ & $21(23.3)$ & $0.024^{\mathrm{a}}$ \\
\hline Age & $30.08 \pm 4.33$ & $29.86 \pm 3.84$ & $0.783^{\mathrm{b}}$ \\
\hline Prepregnant BMI & $21.48 \pm 2.97$ & $21.24 \pm 2.85$ & $0.684^{\mathrm{b}}$ \\
\hline BMI increment & $5.91 \pm 1.90$ & $5.61 \pm 1.67$ & $0.396^{\mathrm{b}}$ \\
\hline Gravidity & $2(1-3)$ & $2(1-3)$ & $0.514^{\mathrm{c}}$ \\
\hline Parity & $1(1-2)$ & $1(1-2)$ & $1.000^{\mathrm{c}}$ \\
\hline Primipara & $28(70.0)$ & $63(70.0)$ & $0.578^{\mathrm{a}}$ \\
\hline Cesarean section & $39(97.5)$ & $82(91.1)$ & $0.173^{\mathrm{a}}$ \\
\hline Gestational weeks (d) & $259.00(247.25-265.75)$ & $261.00(252.75-269.00)$ & $0.368^{\mathrm{c}}$ \\
\hline Male baby & $21(52.5)$ & $44(48.9)$ & $0.425^{\mathrm{a}}$ \\
\hline Neonatal weight $(\mathrm{g})$ & $2415.00(1822.50-3187.50)$ & $3065.00(2352.50-3442.50)$ & $0.007^{\mathrm{c}}$ \\
\hline Education background & $4(3-4)$ & $4(3-4)$ & $0.891^{\mathrm{c}}$ \\
\hline Annual income & $3(2-3)$ & $3(2-3)$ & $0.651^{\mathrm{c}}$ \\
\hline Leakage Index Score & $1(0-2)$ & $0(0-1)$ & $0.208^{\mathrm{c}}$ \\
\hline Pain scale & $1(1-2)$ & $1(1-1)$ & $0.012^{\mathrm{C}}$ \\
\hline Exclusive breastfeeding & $11(27.5)$ & $32(35.5)$ & $0.244^{\mathrm{a}}$ \\
\hline NICU & $11(27.5)$ & $19(21.11)$ & $0.280^{\mathrm{a}}$ \\
\hline
\end{tabular}

${ }^{a}$ The $P$ value is calculated by the chi-squared test. ${ }^{\text {b }}$ The $P$ value is calculated by an independent sample $t$-test. ${ }^{c}$ The $P$ value is calculated by the Kruskal-Wallis test.

TABLE 4: Comparison of EPDS scores in each subgroup.

\begin{tabular}{lcccc}
\hline Variables & Samples $(N)$ & EPDS scores & $t$-test & $P$ value $^{\mathrm{a}}$ \\
\hline Normal & 295 & $7.09 \pm 4.41$ & Reference & Reference \\
Mild PE & 74 & $8.62 \pm 4.35$ & -2.690 & 0.008 \\
Severe PE & 46 & $10.58 \pm 5.41$ & -4.170 & $<0.001$ \\
PE+FGR & 38 & $11.61 \pm 5.29$ & -5.031 & $<0.001$ \\
\hline
\end{tabular}

${ }^{a}$ All of the $P$ values are calculated by an independent sample $t$-test.

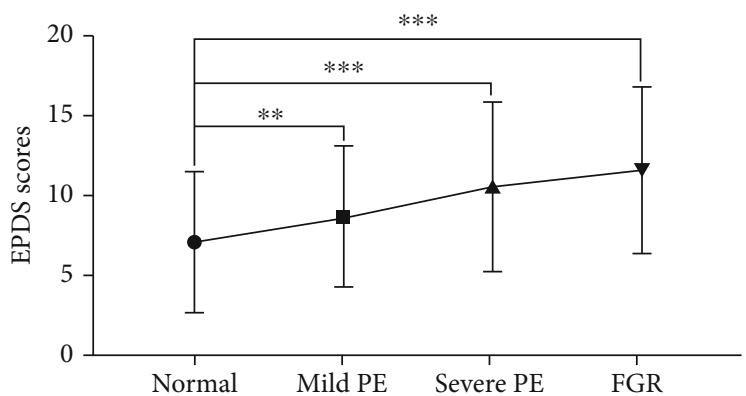

FIGURE 1: The average EPDS score in the normal group was significantly lower than that of the mild $\mathrm{PE}$ subgroup. The $P$ values were shown as ${ }^{* *} P<0.01 ;{ }^{* * *} P<0.001$.

\section{Results}

3.1. Clinical Characteristics between Normal and PE Mothers. A total of $130 \mathrm{PE}$ patients met the inclusion and exclusion criteria. We randomly selected 295 normal women who met the inclusion criteria during the same period. In the PE group, 74 patients were diagnosed with mild PE, the others with severe PE. Clinical characteristics were compared between the normal and PE groups in Table 1. The layer distribution of prepregnancy body mass index (BMI) was significantly different, and PE mothers had a higher BMI than the normal ones $(P=0.005)$. PE patients suffered a much higher rate of cesarean section $(93.08 \%$ vs. 33.22\%) and less gestational age (260 vs. 277 days) (both $P<0.001)$. As a result, the birthweight of the fetuses in the PE group was inferior to the normal one $(2960 v s .3255 \mathrm{~g}, P<0.001)$. In terms of feeding, infant formula was more frequently used in the $\mathrm{PE}$ group, no wonder the exclusive breastfeeding rate was lower (33.08\% vs. 57.63\%, $P<0.001)$. However, other clinical characteristics were not significantly different between groups, such as BMI increase, gravidity, parity, percentage of primiparas, sex of the fetus, educational background, annual family income, and scores of the Leakage Index Questionnaire and pain scale.

3.2. Clinical Characteristics between $P P D$ and Non-PPD Groups. All participants were asked to finish EPDS, and the scores were compared between the normal and PE groups. No differences in clinical characteristics were found between PPD and non-PPD mothers in the normal group (Table 2). However, in the PE group, mothers who had babies with FGR and low neonatal weight tended to develop PPD $(P=0.024$ and $P=0.007$; Table 3$)$. Postpartum pain was another high-risk factor for $\mathrm{PPD}$ in the $\mathrm{PE}$ group $(P=0.012)$. Unlike in the $\mathrm{PE}$ group, the scores of the pain scale showed no difference between PPD and non-PPD women in the normal group $(P=0.209)$. 
TABLE 5: Comparison of characteristics between two different delivery modes in normal women.

\begin{tabular}{lccc}
\hline Variables & C-sections $(N=98)(\%)$ & Vaginal delivery $(N=197)(\%)$ & $P$ value \\
\hline Age & $29.65 \pm 3.88$ & $28.52 \pm 3.43$ & $0.011^{\mathrm{b}}$ \\
Prepregnant BMI & $20.91 \pm 2.47$ & $20.54 \pm 2.41$ & $0.239^{\mathrm{b}}$ \\
BMI increment & $5.87 \pm 1.66$ & $5.70 \pm 1.59$ & $0.381^{\mathrm{b}}$ \\
Gravidity & $2(1-3)$ & $1(1-2)$ & $0.257^{\mathrm{c}}$ \\
Parity & $1(1-2)$ & $146(74.11)$ & $0.270^{\mathrm{c}}$ \\
Primipara & $67(68.37)$ & $277(272-282)$ \\
Gestational weeks (d) & $276(272-282)$ & $113(57.36)$ \\
Male baby & $54(55.10)$ & $3260.0(3020.0-3535.0)$ \\
Neonatal weight (g) & $3252.5(3050.0-3600.0)$ & $4(3-4)$ & $0.335^{\mathrm{a}}$ \\
Education background & $4(3-4)$ & $3(2-3)$ & $0.635^{\mathrm{c}}$ \\
Annual income & $2(2-3)$ & $1(0-2)$ & $0.803^{\mathrm{a}}$ \\
Leakage Index & $0(0-1)$ & $1(1-1)$ & $0.275^{\mathrm{c}}$ \\
Pain scale & $1(1-1)$ & $116(58.88)$ & $0.120^{\mathrm{c}}$ \\
Exclusive breastfeeding & $54(55.10)$ & $7.27 \pm 4.41$ & $0.024^{\mathrm{c}}$ \\
EPDS score & $6.74 \pm 4.42$ & $30(15.23)$ & $0.001^{\mathrm{c}}$ \\
PPD & $13(13.27)$ & $0.617^{\mathrm{a}}$ \\
\hline
\end{tabular}

${ }^{\mathrm{a}}$ The $P$ value is calculated by a chi-squared test. ${ }^{\mathrm{b}}$ The $P$ value is calculated by an independent sample $t$-test. ${ }^{\mathrm{c}}$ The $P$ value is calculated by the Kruskal-Wallis test.

TABLE 6: Comparison of baseline characteristics in normal and PE women who suffered cesarean section.

\begin{tabular}{lccc}
\hline Variables & PE $(N=121)(\%)$ & Normal $(N=98)(\%)$ & $P$ value \\
\hline Age & $30.02 \pm 3.81$ & $29.65 \pm 3.88$ & $0.488^{\mathrm{b}}$ \\
Prepregnant BMI & $21.27 \pm 2.87$ & $20.91 \pm 2.47$ & $0.316^{\mathrm{b}}$ \\
BMI increment & $5.74 \pm 1.76$ & $5.87 \pm 1.66$ & $0.576^{\mathrm{b}}$ \\
Gravidity & $2(1-3)$ & $2(1-3)$ & $0.240^{\mathrm{c}}$ \\
Parity & $1(1-2)$ & $1(1-2)$ & $0.623^{\mathrm{c}}$ \\
Primipara & $85(70.25)$ & $276(272-282)$ & $0.770^{\mathrm{a}}$ \\
Gestational weeks (d) & $260(251-269)$ & $54(55.10)$ & $3252.5(3050.0-3600.0)$ \\
Male baby & $60(49.59)$ & $4(3-4)$ & $<0.001^{\mathrm{c}}$ \\
Neonatal weight (g) & $2960.0(2170.0-3400.0)$ & $2(2-3)$ & $0.497^{\mathrm{a}}$ \\
Education background & $4(3-4)$ & $0(0-1)$ & $0.141^{\mathrm{c}}$ \\
Annual income & $3(2-3)$ & $1(1-1)$ & $0.172^{\mathrm{c}}$ \\
Leakage Index & $0(0-1)$ & $54(55.10)$ & $0.073^{\mathrm{c}}$ \\
Pain scale & $1(1-1)$ & $6.74 \pm 4.42$ & $0.240^{\mathrm{c}}$ \\
Exclusive breastfeeding & $40(33.06)$ & $13(13.27)$ & $0.002^{\mathrm{a}}$ \\
EPDS score & $9.54 \pm 4.80$ & $39(32.23)$ & $<0.001^{\mathrm{b}}$ \\
PPD & $0.001^{\mathrm{a}}$ \\
\hline
\end{tabular}

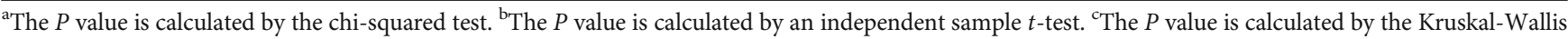
test.

3.3. Severe PE and FGR Women Were Inclined to Develop Higher EPDS Scores. We tried to explore the associations between PPD and PE. The average EPDS score in the normal group was significantly lower than that of the mild PE subgroup ( $7.09 \pm 4.41$ vs. $8.62 \pm 4.35, P=0.008$; Table 4 and Figure 1). In the severe PE subgroup, the average EPDS score was even worse $(10.58 \pm 5.41)$, indicating that most of the severe PE patients developed PPD. Furthermore, $38 \mathrm{PE}$ mothers were complicated with FGR and got the highest EPDS scores among the subgroups $(11.61 \pm 5.29, P<0.001)$.
3.4. Rather than Cesarean Section, PE Showed a Direct Tendency on PPD Development. There was a higher cesarean section rate among $\mathrm{PE}$ patients than normal women $(93.08 \%$ vs. $33.22 \%)$. To determine the effect of the cesarean section on PPD development, we compared the EPDS scores and PPD incidence between $\mathrm{C}$-section and vaginal delivery in the normal group. There was not any difference in the EPDS score $(6.74 \pm 4.42$ vs. $7.27 \pm 4.41, P=0.337)$ and PPD incidence $(13.27 \%$ vs. $15.32 \%, P=0.728)$ between the two delivery modes (Table 5). Interestingly, when comparing the 
TABLE 7: Comparison of the incidence of PPD between normal and PE women.

\begin{tabular}{lccc}
\hline Variables & PPD (\%) & Non-PPD (\%) & $P$ value $^{\mathrm{a}}$ \\
\hline Normal $(N=295)$ & $43(14.58)$ & $252(85.42)$ & Reference \\
Total PE $(N=130)$ & $40(30.77)$ & $90(69.23)$ & $<0.001$ \\
Mild PE $(N=74)^{\#}$ & $20(27.03)$ & $54(72.97)$ & 0.014 \\
Severe PE $(N=46)$ & $16(36.96)$ & $30(63.04)$ & 0.002 \\
PE+FGR $(N=38)$ & $17(44.74)$ & $21(52.26)$ & $<0.001$ \\
NICU $(N=30)$ & $11(36.66)$ & $19(63.33)$ & 0.004 \\
PE+preterm $(N=61)$ & $20(32.79)$ & $41(67.21)$ & 0.001 \\
\hline
\end{tabular}

${ }^{\text {a All of the } P \text { values are calculated by the chi-squared test. }{ }^{*} \text { In this group, } 10}$ subjects with mild PE but with FGR were excluded.

normal and PE groups suffering from cesarean section, it was found that both the EPDS $(9.54 \pm 4.80 v s .6 .74 \pm 4.42, P<$ $0.001)$ score and PPD incidence $(32.23 \%$ vs. $13.27 \%, P=$ $0.001)$ were much higher among the PE group than the normal group (Table 6). It could be inferred that it was not cesarean section but PE that directly increased the risk of PPD.

3.5. Much Higher Screening of PPD in PE Mothers than the Normal Ones. We compared the rate of positive screening of PPD in each subgroup (Table 7). Totally, 83 people were screened positive for $\mathrm{PPD}$, while the remaining 342 were negative. The incidence of PPD was $14.58 \%$ in the normal group, whereas the rate was much higher among PE mothers. About $30.77 \%$ of women in the PE group met the diagnostic criterion for PPD. Furthermore, the incidences of PPD dramatically increased with the severity of $\mathrm{PE}$ and its complications. For instance, the incidences of PPD in the mild PE and severe PE subgroups were $27.03 \%$ and $36.96 \%$, respectively, which were significantly higher than that of the normal mothers $(14.58 \%, P=0.014$ and $P=0.002)$.

We also tried to explore the associations between $\mathrm{PE}$ complications and PPD development. In the PE+FGR subgroup, the incidence of PPD was the highest among all the subgroups (44.74\%). Thirty newborns were extremely weak and had to be sent to the neonatal intensive care unit (NICU). Obviously, when the babies were sent to NICU, their mothers tended to develop PPD. PPD incidence among these mothers increased dramatically (36.66\%), which was extremely high. Preterm, one of the common complications in PE, occurred in almost half of PE mothers (61 of 130). $\mathrm{PPD}$ occurrence was $32.79 \%$ in the $\mathrm{PE}+$ preterm subgroup.

3.6. Independent Risk Factors for PPD. Then, multiple logistic regression was performed to evaluate the independent risk factors for PPD. With PPD as the dependent variable, PE, severe PE, FGR, and NICU admission were regarded as independent variables individually, while age, BMI, gestational days, baby weight, delivery model, Leakage Index Score, milk-feeding ways, and pain scale were analyzed as confounding factors. Women with mild PE demonstrated 2fold higher odds of PPD (AOR $=2.117,95 \% \mathrm{CI}$ : 1.001 4.479; Table 8). Furthermore, severe PE, FGR, and NICU admission all increased nearly 3 -fold risk for PPD positive screening. These findings indicate that the severity and com- plications of PE will increase the risk of PPD (as shown in Figure 2).

Besides PE, postpartum pain was another independent risk factor for $\mathrm{PPD}(\mathrm{AOR}=1.509,95 \% \mathrm{CI}$ : 1.078-2.114). The effect of breastfeeding on PPD has not been indicated before, but in our study, exclusive breastfeeding seemed not to positively affect the mood of the mothers $(\mathrm{AOR}=0.752,95 \% \mathrm{CI}$ : 0.445-1.270). Pelvic floor muscle recovery has always been a concern among new mothers and can dramatically influence their moods. After evaluating pelvic floor function, we found that the dysfunction of pelvic floor muscles had no negative effect on PPD (AOR = 1.137, 95\% CI: 0.952-1.358). Moreover, we observed that there were no correlations between the cesarean section and PPD (AOR = 1.177, 95\% CI: 0.620-2.232).

\section{Discussion}

Among the general population, hypertension has already been proved to be an independent risk factor for depressive disorder [23]. Hypertension increased 1.12-fold of developing depression among 6,237 old Chinese adults [24]. For pregnant women, few studies were exploring the connection between PE and PPD. To our knowledge, this is the first retrospective cohort study to clarify the associations of the severity and complications of PE with PPD in the Chinese population. The number of cases (425) in our trial is the largest among the existing relevant studies.

As reported, PPD occurred in $20.5 \%$ of PE patients in Tanzanian and in about $21 \%$ of PE mothers in Greek [11, 25]. In our study, the percentage of positive screening for PPD in the PE group was even higher (30.77\%). Besides PE, its complications could also increase the risk of PPD. Similar to our findings, Hoedjes et al. discovered that the prevalence of PPD was 23\% in mild PE patients and $44 \%$ in severe PE [26]. These studies suggest that PE affects PPD strongly.

For PE mothers, besides the unfavorable experience of hypertension, other conditions such as additional costs and concerns of the newborns with complications also increase mothers' psychological burden [27, 28]. The outcomes of infants play an important role in PPD development among severe PE patients [26]. This conclusion was confirmed in our study, especially for growth-restricted babies. A study reported that the prevalence of PPD among the FGR family was $48.2 \%$, which was similar to our result (44.74\%) [27]. 38\% of mothers experienced significant depressive symptoms when their babies were sent to NICU [29]. These studies mentioned that baby conditions and financial problems may be two of the most risk factors for PPD. In our clinical trial, mothers were asked, "What most upsets you?" The majority of mothers told us that they were bothered most by the poor outcomes of their babies and NICU admission. In our study, 30 neonates were admitted to NICU. Notably, the incidence of PPD among these mothers was very high (36.6\%). Many randomized controlled trials have implicated that insufficient contact with babies will increase the odds of PPD [30-32]. Therefore, clinical healthcare workers should provide psychological supplies to mothers with NICU babies. The financial problem was the second problem: seven of them received lower annual income (less than $80 \mathrm{k} \mathrm{RMB}$ 
TABLE 8: Multivariable logistic regression analysis for PPD in PE and normal patients.

\begin{tabular}{|c|c|c|}
\hline Variables & OR $(95 \% \mathrm{CI})$ & Adjusted OR $(95 \% \mathrm{CI})^{\mathrm{a}}$ \\
\hline Age & $0.987(0.925-1.052)$ & \\
\hline Prepregnant BMI & $1.006(0.917-1.103)$ & \\
\hline Gestational weeks (d) & $0.985(0.851-1.139)$ & \\
\hline Baby weight & $0.999(0.999-1.000)$ & \\
\hline Leakage Index & $1.137(0.952-1.358)$ & \\
\hline Educational background & $0.953(0.731-1.241)$ & \\
\hline Annual income & $1.020(0.741-1.403)$ & \\
\hline Cesarean section & $1.758(1.074-2.887)$ & $1.177(0.620-2.232)^{\#}$ \\
\hline Exclusive breastfeeding & $0.558(0.342-0.911)$ & $0.752(0.445-1.270)^{\# \#}$ \\
\hline Pain scale & $1.581(1.151-2.174)$ & $1.509(1.078-2.114)^{*}$ \\
\hline Mild PE & $2.171(1.184-3.981)$ & $2.117(1.001-4.479)^{*}$ \\
\hline Severe PE & $3.126(1.527-6.216)$ & $2.759(1.206-6.315)^{*}$ \\
\hline FGR & $4.744(2.317-9.713)$ & $3.450(1.596-7.458)^{*}$ \\
\hline NICU & $2.597(1.184-5.696)$ & $2.809(1.258-6.270)^{*}$ \\
\hline
\end{tabular}

${ }^{a}$ The adjusted ORs were calculated by multifactor logistic regression models. ${ }^{\#}$ Adjusted factors: age, BMI, and PE. ${ }^{\# \#}$ Adjusted factors: age, BMI, PE, and cesarean section. *Adjusted factors: age, BMI, cesarean section, uroclepsia, breastfeeding, educational background, and annual income.

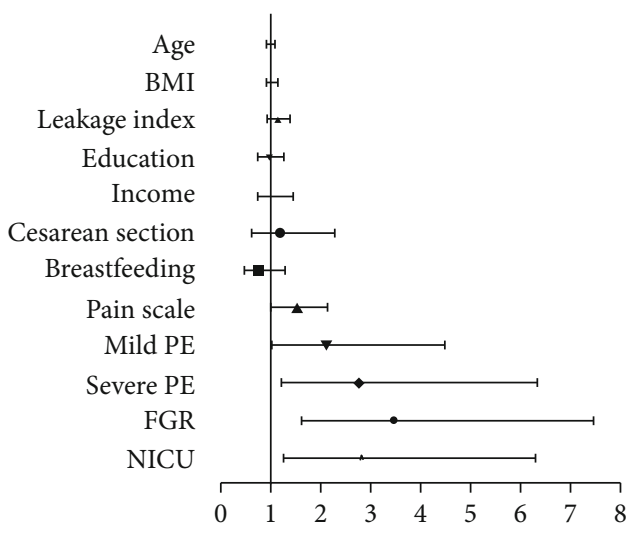

FIgURE 2: The odds ratio of PPD for each characteristic. The line segment represents the odds ratio and $95 \%$ confidence interval for each variable. It shows that the pain scale, mild PE, severe PE, FGR, and NICU were risk factors with OR and 95\%CI > 1 .

per year); they felt huge burdens on children's hospital expenses.

Whether the cesarean section will increase the risk of PPD is still controversial. In China, some healthy pregnant women would like to choose a cesarean section due to social-psychological factors. In this research, mothers with PE preferred to have a cesarean section to avoid possible adverse outcomes. This can explain why the rate of operative delivery in China among PE patients is so high. First of all, to figure out the effect of the cesarean section for PPD, we compared delivery models among normal women. Patel et al. demonstrated that operative delivery would not increase the incidence of PPD in 14,633 women [33]. A meta-analysis in 2017 also reported that elective cesarean section would not significantly exacerbate the odds of PPD (AOR: 1.15, 95\% CI: 0.92-1.43) [34]. In our study, there was not any difference in the EPDS score and PPD incidence between the two delivery models among normal pregnant populations.

However, in the PE group, we found that both EPDS score and PPD incidence were much higher in mothers suffering from the operation. It could be inferred that $\mathrm{PE}$ directly increased the risk of PPD rather than cesarean section. Then, we applied subgroup analysis to find the reason. In the PE+FGR subgroup, the incidence of PPD was the highest among all the subgroups. Obviously, mothers tended to show anxiety when babies were sent to NICU. Another common complication is preterm. Almost half of PE mothers occurred preterm. As expected, mothers in the PE+preterm group experienced higher psychological distress than others. Weigl et al. pointed out that new mothers of preterm infants exhibited higher scores of depression, anxiety, and stress than parents of term infants [35]. Preterm mothers showed lower levels of estradiol, progesterone, and prolactin, as well as a heightened postawakening cortisol response, compared to term mothers. These results are consistent with our findings.

Postpartum pain, urinary incontinence, and feeding methods were also evaluated in the regression model. Postpartum pain was an independent risk factor for PPD, increasing the odds by 1.5 -fold. A few trials showed that untreated pain is associated with a risk of PPD $[36,37]$. The usage of painkillers can help decrease the incidence of $P P D$ in some cases $[38,39]$. Our study implied postpartum pain as another risk factor for PPD in the PE group. This was probably because $\mathrm{PE}$ mothers suffered more postpartum pain from the operation. Therefore, it is reasonable to use painkillers for PE mothers during the postnatal period.

Hullfish et al. have demonstrated a correlation between urinary incontinence and PPD [40], but Doering et al. showed no such connection [41]. In our study, there was no significant result about urinary incontinence in PPD development. Nonetheless, more authoritative urinary incontinence scales need to be tested in the future. 
Nonbreastfeeding was regarded as a risk factor for PPD in many cases [42, 43]. But in our study, exclusive breastfeeding did not help decrease the incidence of PPD.

Although the connection between PE and PPD is still unclear, some mechanisms, such as clinical symptoms, inflammation, and genetic changes, have been used as hypotheses for the reason between PE and PPD. The pathogenesis for PE, a placenta disease, can be explained by the "two-stage theory" [44]. At the first stage, vascular remodeling disorders of uterine spiral arterioles caused by multiple factors result in "superficial placental implantation" and ultimately cause insufficient placental perfusion and impairment of placental function. In the second stage, the ischemic placenta will experience oxidative stress and release inflammatory factors, such as IL-6, leading to systemic endothelial dysfunction. Therefore, PE patients often have excessive inflammatory factors in blood circulation [45-48]. For example, abnormally elevated C-reactive protein (CRP) and tumor necrosis factor- (TNF-) $\alpha$ are detected in the serum of $\mathrm{PE}$ mothers, resulting in vascular remodeling dysfunction of the placenta [49]. Consistently, like PE, inflammatory biomarkers also take part in PPD development [50]. Studies confirm that increased IL-6 and TNF- $\alpha$ levels during the perinatal period can intensify the risk of PPD [51-53]. Based on these studies, we speculate that inflammatory cytokines are released by the dysfunctional placenta in PE mothers, finally leading to the development of PPD [48, 53, 54]. Our further research will pay more attention to these inflammatory cytokines.

\section{Limitations}

We must admit that there are some limitations in our study. As a retrospective study, it suffered from bias and case limitations. Firstly, patients were recruited from 2 hospitals, and the local bias may be relatively reduced, but there is still a need for a study involving multiple centers. Secondly, it was hard to control the operation rate in the PE group, although this delivery mode was not found to be a risk factor in our regression model. Thirdly, EPDS was a preliminary screening tool, not the gold standard for the diagnosis of PPD. In the future, we would like to initiate larger randomized controlled trials and more in-depth mechanistic studies.

\section{Conclusions}

PE can be an independent risk factor for PPD. Moreover, its severity and complications exacerbate the development of PPD. Severe PE, FGR, and NICU admission all increased nearly 3-fold risk for PPD-positive screening. Patients with $P E$ should be offered suitable interventions, such as pain management, more cognitive-behavioral therapies (CBT), and interpersonal psychotherapies (IPT) to prevent the development of PPD.

\section{Data Availability}

The data used and analyzed during the current study are available from the corresponding authors on reasonable request.

\section{Ethical Approval}

The study was approved by the ethics committee of the First Affiliated Hospital of Chongqing Medical University on 1 December 2019 (No. 20198101).

\section{Consent}

Electronic informed consent was obtained before completing the questionnaire.

\section{Disclosure}

This manuscript has been submitted as a preprint; here is the website link: https://www.researchgate.net/publication/ 343953077_Preeclampsia_and_Its_Complications_

Exacerbate_Development_of_Postpartum_Depression_A_ Retrospective_Cohort_Study. Some details have been modified.

\section{Conflicts of Interest}

None is declared. Completed disclosure of interest forms are available to view online as supporting information.

\section{Acknowledgments}

The authors would like to acknowledge support from the "111 program" of the Ministry of Education of PRC and the State Administration of Foreign Experts Affairs PRC. This work was financially supported by the National Key Research and Development Program of Reproductive Health \& Major Birth Defects Control and Prevention (No. 2016YFC1000407), the National Natural Science Foundation of China for Youth (81601304), the Key Program of International Cooperation of NSFC (81520108013), the Ph.D. Programs Foundation of the Ministry of Education of China (2013550311003), and the General Program of the National Natural Science Foundation of China (81471472).

\section{Supplementary Materials}

Figure S1: the questionnaires were used in the study, including EPDS, Leakage Index Questionnaire, and pain scale. (Supplementary Materials)

\section{References}

[1] E. Molyneaux, L. A. Telesia, C. Henshaw et al., "Antidepressants for preventing postnatal depression," The Cochrane Database of Systematic Reviews, vol. 4, article CD004363, 2018.

[2] J. Hahn-Holbrook, T. Cornwell-Hinrichs, and I. Anaya, "Economic and health predictors of national postpartum depression prevalence: a systematic review, meta-analysis, and meta-regression of 291 studies from 56 countries," Frontiers in Psychiatry, vol. 8, 2018.

[3] M. Nakano, A. Sourander, T. Luntamo, R. Chudal, N. Skokauskas, and H. Kaneko, "Early risk factors for postpartum depression: a longitudinal Japanese population-based study," Journal of Affective Disorders, vol. 269, pp. 148-153, 2020. 
[4] S.-C. Weng, J.-C. Chang, M.-K. Yeh, S.-M. Wang, and Y.$\mathrm{H}$. Chen, "Factors influencing attempted and completed suicide in postnatal women: a population-based study in Taiwan," Scientific Reports, vol. 6, no. 1, 2016.

[5] M. K. Krischer, M. H. Stone, K. Sevecke, and E. M. Steinmeyer, "Motives for maternal filicide: results from a study with female forensic patients," International Journal of Law and Psychiatry, vol. 30, no. 3, pp. 191-200, 2007.

[6] S. R. Brand and P. A. Brennan, "Impact of antenatal and postpartum maternal mental illness: how are the children?," Clinical Obstetrics and Gynecology, vol. 52, no. 3, pp. 441-455, 2009.

[7] B. Avan, L. M. Richter, P. G. Ramchandani, S. A. Norris, and A. Stein, "Maternal postnatal depression and children's growth and behaviour during the early years of life: exploring the interaction between physical and mental health," Archives of Disease in Childhood, vol. 95, no. 9, pp. 690-695, 2010.

[8] G. Hammerton, S. Zammit, A. Thapar, and S. Collishaw, "Explaining risk for suicidal ideation in adolescent offspring of mothers with depression," Psychological Medicine, vol. 46, no. 2, pp. 265-275, 2016.

[9] T. Azale, A. Fekadu, and C. Hanlon, "Postpartum depressive symptoms in the context of high social adversity and reproductive health threats: a population-based study," International Journal of Mental Health Systems, vol. 12, no. 1, 2018.

[10] J. N. Baumgartner, A. Parcesepe, Y. G. Mekuria et al., "Correlates of postpartum common mental disorders: results from a population-based study in Amhara region, Ethiopia," Archives of Women's Mental Health, vol. 19, no. 5, pp. 937-942, 2016.

[11] B. Mbarak, C. Kilewo, S. Kuganda, and B. F. Sunguya, "Postpartum depression among women with pre-eclampsia and eclampsia in Tanzania; a call for integrative intervention," BMC Pregnancy and Childbirth, vol. 19, no. 1, 2019.

[12] M. A. Brown, L. A. Magee, L. C. Kenny et al., "Hypertensive disorders of pregnancy: ISSHP classification, diagnosis, and management recommendations for international practice," Hypertension, vol. 72, no. 1, pp. 24-43, 2018.

[13] G. J. Burton, C. W. Redman, J. M. Roberts, and A. Moffett, "Pre-eclampsia: pathophysiology and clinical implications," $B M J$, vol. 366, 2019.

[14] E. A. Phipps, R. Thadhani, T. Benzing, and S. A. Karumanchi, "Pre-eclampsia: pathogenesis, novel diagnostics and therapies," Nature Reviews. Nephrology, vol. 15, no. 5, pp. 275289, 2019.

[15] L. Chen, X. Wang, Q. Ding, N. Shan, and H. Qi, “Development of postpartum depression in pregnant women with preeclampsia: a retrospective study," BioMed Research International, vol. 2019, Article ID 9601476, 7 pages, 2019.

[16] C. W. Swenson, J. A. DePorre, J. K. Haefner, M. B. Berger, and D. E. Fenner, "Postpartum depression screening and pelvic floor symptoms among women referred to a specialty postpartum perineal clinic," American Journal of Obstetrics and Gynecology, vol. 218, no. 3, pp. 335.e1-335.e6, 2018.

[17] D. Shen, M. Hasegawa-Moriyama, K. Ishida, S. Fuseya, S. Tanaka, and M. Kawamata, "Acute postoperative pain is correlated with the early onset of postpartum depression after cesarean section: a retrospective cohort study," Journal of Anesthesia, vol. 34, no. 4, pp. 607-612, 2020.

[18] ACOG, "ACOG committee opinion no. 757: screening for perinatal depression," Obstetrics and Gynecology, vol. 132, no. 5, pp. e208-e212, 2018.
[19] J. L. Cox, J. M. Holden, and R. Sagovsky, "Detection of postnatal depression," The British Journal of Psychiatry, vol. 150, no. 6, pp. 782-786, 1987.

[20] W. V. Bobo and B. P. Yawn, "Concise review for physicians and other clinicians: postpartum depression," Mayo Clinic Proceedings, vol. 89, no. 6, pp. 835-844, 2014.

[21] E. O'Connor, R. C. Rossom, M. Henninger, H. C. Groom, and B. U. Burda, "Primary care screening for and treatment of depression in pregnant and postpartum women: evidence report and systematic review for the US preventive services task force," JAMA, vol. 315, no. 4, pp. 388-406, 2016.

[22] ACOG, "ACOG practice bulletin no. 202 summary: gestational hypertension and preeclampsia," Obstetrics and Gynecology, vol. 1, no. 6, pp. 211-214, 133.

[23] J. Xue, S. Chen, H. R. Bogner, W. Tang, L. Li, and Y. Conwell, "The prevalence of depressive symptoms among older patients with hypertension in rural China," International Journal of Geriatric Psychiatry, vol. 32, no. 12, pp. 1411-1417, 2017.

[24] Y. Jin, Y. Luo, and P. He, "Hypertension, socioeconomic status and depressive symptoms in Chinese middle- aged and older adults: Findings from the China health and retirement longitudinal study," Journal of Affective Disorders, vol. 252, pp. 237-244, 2019.

[25] K. Koutra, M. Vassilaki, V. Georgiou et al., "Pregnancy, perinatal and postpartum complications as determinants of postpartum depression: the Rhea mother-child cohort in Crete, Greece," Epidemiology and Psychiatric Sciences, vol. 27, no. 3, pp. 244-255, 2018.

[26] M. Hoedjes, D. Berks, I. Vogel et al., "Postpartum depression after mild and severe preeclampsia," Journal of Women's Health, vol. 20, no. 10, pp. 1535-1542, 2011.

[27] N. Helle, C. Barkmann, S. Ehrhardt, A. von der Wense, Y. Nestoriuc, and C. Bindt, "Postpartum anxiety and adjustment disorders in parents of infants with very low birth weight: crosssectional results from a controlled multicentre cohort study," Journal of Affective Disorders, vol. 194, pp. 128-134, 2016.

[28] L. Garfield, D. Holditch-Davis, C. S. Carter et al., "Risk factors for postpartum depressive symptoms in low-income women with very low-birth-weight infants," Advances in Neonatal Care, vol. 15, no. 1, pp. E3-E8, 2015.

[29] A. Alkozei, E. McMahon, and A. Lahav, "Stress levels and depressive symptoms in NICU mothers in the early postpartum period," The Journal of Maternal-Fetal \& Neonatal Medicine, vol. 27, no. 17, pp. 1738-1743, 2014.

[30] K. Mehler, E. Hucklenbruch-Rother, P. Trautmann-Villalba, I. Becker, B. Roth, and A. Kribs, "Delivery room skin-to-skin contact for preterm infants-a randomized clinical trial," Acta Paediatrica, vol. 109, no. 3, pp. 518-526, 2020.

[31] N. V. Scime, A. G. Gavarkovs, and K. H. Chaput, "The effect of skin-to-skin care on postpartum depression among mothers of preterm or low birthweight infants: a systematic review and meta-analysis," Journal of Affective Disorders, vol. 253, pp. 376-384, 2019.

[32] K. H. M. Cooijmans, R. Beijers, A. C. Rovers, and C. de Weerth, "Effectiveness of skin-to-skin contact versus care-asusual in mothers and their full-term infants: study protocol for a parallel-group randomized controlled trial," BMC Pediatrics, vol. 17, no. 1, 2017.

[33] R. R. Patel, D. J. Murphy, and T. J. Peters, "Operative delivery and postnatal depression: a cohort study," BMJ, vol. 330, no. 7496, 2005. 
[34] H. Xu, Y. Ding, Y. Ma, X. Xin, and D. Zhang, "Cesarean section and risk of postpartum depression: a meta-analysis," Journal of Psychosomatic Research, vol. 97, pp. 118-126, 2017.

[35] T. Weigl, N. Schneider, A. Stein, U. Felderhoff-Müser, M. Schedlowski, and H. Engler, "Postpartal affective and endocrine differences between parents of preterm and full-term infants," Frontiers in Psychiatry, vol. 11, 2020.

[36] ACOG, "ACOG Committee opinion no. 742 summary: postpartum pain management," Obstetrics and Gynecology, vol. 132, pp. 252-253, 2018.

[37] J.-H. Ma, S.-Y. Wang, H.-Y. Yu et al., "Prophylactic use of ketamine reduces postpartum depression in Chinese women undergoing cesarean section ${ }^{\text {is }}$," Psychiatry Research, vol. 279, pp. 252-258, 2019.

[38] H.-Y. Yu, S.-Y. Wang, C.-X. Quan et al., "Dexmedetomidine alleviates postpartum depressive symptoms following cesarean section in Chinese women: a randomized placebo-controlled study," Pharmacotherapy: The Journal of Human Pharmacology and Drug, vol. 39, no. 10, pp. 994-1004, 2019.

[39] S. Orbach-Zinger, R. Landau, A. B. Harousch et al., "The relationship between women's intention to request a labor epidural analgesia, actually delivering with labor epidural analgesia, and postpartum depression at 6 weeks: a prospective observational study," Anesthesia and Analgesia, vol. 126, no. 5, pp. 1590-1597, 2018.

[40] K. L. Hullfish, D. E. Fenner, S. A. Sorser, J. Visger, A. Clayton, and W. D. Steers, "Postpartum depression, urge urinary incontinence, and overactive bladder syndrome: is there an association?," International Urogynecology Journal and Pelvic Floor Dysfunction, vol. 18, no. 10, pp. 1121-1126, 2007.

[41] A. D. Doering, A. F. Hundley, C. O. Hudson, J. A. Benedict, C. D. Lynch, and J. A. Schaffir, "Is there an association between bothersome urinary symptoms and postpartum depression?," Female Pelvic Medicine \& Reconstructive Surgery, vol. 25, no. 4, pp. 323-327, 2019.

[42] T. Sha, X. Gao, C. Chen et al., "A prospective study of maternal postnatal depressive symptoms with infant-feeding practices in a Chinese birth cohort," BMC Pregnancy and Childbirth, vol. 19, no. 1, 2019.

[43] E. L. Stark, J. Shim, C. M. Ross, and E. S. Miller, "The association between positive antenatal depression screening and breastfeeding initiation and continuation," American Journal of Perinatology, vol. 38, no. 2, pp. 171-175, 2021.

[44] S. Rana, E. Lemoine, J. P. Granger, and S. A. Karumanchi, "Preeclampsia: pathophysiology, challenges, and perspectives," Circulation Research, vol. 124, no. 7, pp. 1094-1112, 2019.

[45] B. W. J. Mol, C. T. Roberts, S. Thangaratinam, L. A. Magee, C. J. M. de Groot, and G. J. Hofmeyr, "Pre-eclampsia," Lancet, vol. 387, no. 10022, pp. 999-1011, 2016.

[46] A. Erlebacher, "Immunology of the maternal-fetal interface," Annual Review of Immunology, vol. 31, no. 1, pp. 387-411, 2013.

[47] S. Goulopoulou and S. T. Davidge, "Molecular mechanisms of maternal vascular dysfunction in preeclampsia," Trends in Molecular Medicine, vol. 21, no. 2, pp. 88-97, 2015.

[48] T. Chaiworapongsa, P. Chaemsaithong, L. Yeo, and R. Romero, "Pre-eclampsia part 1: current understanding of its pathophysiology," Nature Reviews. Nephrology, vol. 10, no. 8 , pp. $466-480,2014$.
[49] E. Laresgoiti-Servitje, N. Gomez-Lopez, and D. M. Olson, “An immunological insight into the origins of pre-eclampsia," Human Reproduction Update, vol. 16, no. 5, pp. 510-524, 2010.

[50] M. Lambert and F. Gressier, "Biomarqueurs de L'inflammation et Dépression du Post-Partum. Une Revue Systématique De la Littérature," Canadian Journal of Psychiatry, vol. 64, no. 7, 2019.

[51] H. Liu, Y. Zhang, Y. Gao, and Z. Zhang, "Elevated levels of HsCRP and IL-6 after delivery are associated with depression during the 6 months post partum," Psychiatry Research, vol. 243 , pp. 43-48, 2016.

[52] E. S. Miller, D. Hoxha, E. Pinheiro, W. A. Grobman, and K. L. Wisner, "The association of serum C-reactive protein with the occurrence and course of postpartum depression," Archives of Women's Mental Health, vol. 22, no. 1, pp. 129-132, 2019.

[53] K. K. Ferguson, J. D. Meeker, T. F. McElrath, B. Mukherjee, and D. E. Cantonwine, "Repeated measures of inflammation and oxidative stress biomarkers in preeclamptic and normotensive pregnancies," American Journal of Obstetrics and Gynecology, vol. 216, no. 5, pp. 527.e1-527.e9, 2017.

[54] A. C. Harmon, D. C. Cornelius, L. M. Amaral et al., "The role of inflammation in the pathology of preeclampsia," Clinical Science, vol. 130, no. 6, pp. 409-419, 2016. 